\title{
Die Wissenschaftlichen Bibliotheken der Schweiz zwischen Literatur- und Informationsversorgung, Dienstleistungen für Studium, Forschung und Bildung und kulturellem Auftrag: eine Standortbestimmung 2018
}

\section{Einleitung}

In diesem Band aus Anlass des Rücktritts von Susanna Bliggenstorfer vom Amt der Direktorin der Zentralbibliothek Zürich präsentieren die Autorinnen und Autoren eine repräsentative Auswahl aktueller Projekte aus der Schweizer Bibliothekslandschaft mit hohem Innovationspotenzial und stellen sie in ihren wissenschafts- und förderpolitischen Kontext. Exemplarische Gebäude- und Raumentwicklungen an Schweizer Bibliotheken werden präsentiert und verdeutlichen die bleibende, ja zunehmende Bedeutung des Orts Bibliothek im Umfeld von Stadt und Hochschule. Mit dem Thema Forschungsdaten wird ein für die Bibliotheken neues Handlungsfeld in den Fokus gerückt, bei dem Rollen und Aufgaben derzeit erkundet werden, und mit den national ausgerichteten Plattformen zu historischen Materialien, Zeitungen, Zeitschriften und Bildern tritt der Zusammenhang von Objektdigitalisierung, Archivauftrag und Bereitstellung für die Forschung in den Vordergrund. Für die Leserinnen und Leser dieser Festschrift gerade ausserhalb der Schweiz mag es da eingangs von Interesse sein zu erfahren, in welchem grösseren institutionellen und kooperativen Rahmen diese Initiativen stehen. Dieser Beitrag gibt daher einen Überblick über Organisation und institutionelle Gegebenheiten des wissenschaftlichen Bibliothekswesens der Schweiz, präsentiert Akteure und Hintergründe und möchte so dazu anregen, Besonderheiten ebenso wie Vergleichbares zu den Verhältnissen in den umliegenden Ländern zu erkennen und einzuordnen. 


\section{Wissenschaftliche und Öffentliche Bibliotheken - Typologie mit Unschärfen}

Auch wenn die traditionellen Spartengrenzen des Bibliothekswesens in bestimmten Bereichen - etwa der zeitgemässen Interpretation des Bildungsauftrags der Bibliotheken - tendenziell an Bedeutung verlieren und derzeit in der Schweiz auf Ebene der Berufs- und Institutionenverbände eine Integration angestrebt wird, ${ }^{1}$ so ist doch auch für die Schweiz die Dualität von Öffentlichen und Wissenschaftlichen Bibliotheken bestimmend: erstere in der Zuständigkeit der Gemeinden und stark in lokalen Strukturen verankert, letztere in der überwiegenden Trägerschaft von Hochschulen oder Kantonen, nur im Fall der Nationalbibliothek auch in der direkten Verantwortung des Bundes angesiedelt. Über die Öffentlichen Bibliotheken in der Schweiz und ihre aktuelle Entwicklung wird hier nicht gesprochen, der vorliegende Aufsatz wie auch die nachfolgenden Beiträge beschränken sich in ihrer Perspektive auf die Welt der Wissenschaftlichen Bibliotheken.

Wobei die Trennung auf den zweiten Blick tatsächlich doch nicht überall so klar zu ziehen ist: Gerade in den kleineren Kantonen der Schweiz findet man nicht selten eine Verbindung der Kantonsbibliothek mit der Stadt- und Gemeindebibliothek. Während die in der Regel im frühen 19. Jahrhundert gegründeten Kantonsbibliotheken über historische Sammlungen mit archivischem Charakter verfügen und oft ältere Einrichtungen (etwa in der Folge der Säkularisierung Kloster- und Stiftsbibliotheken) aufgenommen haben, bieten sie in der Funktion als Gemeinde- und Regionalbibliothek (auch) nicht-wissenschaftliche Literatur für Bildung, Arbeitsleben und Freizeit an, sind Ort an eine breite Öffentlichkeit adressierter kultureller Aktivitäten und engagieren sich beispielsweise im Feld der Leseförderung für Kinder und Jugendliche. ${ }^{2}$ Man wird diesen Typus, der früher in der deutschsprachigen Schweiz in einem Schichtenmodell der Biblio-

1 Die beiden (jeweils auf nationaler Ebene agierenden) Verbände Bibliothek Information Schweiz (BIS) und Schweizerische Arbeitsgemeinschaft der allgemeinen öffentlichen Bibliotheken/Communauté de travail des bibliothèques suisses de lecture publique (SAB/CLP) sollen gemäss einer Initiative beider Vorstände im Jahr 2018 zum gemeinsamen Verband Bibliosuisse verschmelzen. Vgl. dazu den Beitrag „Bibliotheksverband“ von Herbert Staub in diesem Band.

2 Exemplarisch seien als grössere Kantonsbibliotheken dieses Typs die Biblioteca cantonale del Ticino mit ihren drei Standorten in Lugano, Bellinzona und Mendrisio, die Médiathèque du Valais, die Kantonsbibliothek Vadiana in St. Gallen, die Stadtbibliothek Schaffhausen oder die Thurgauer Kantonsbibliothek in Frauenfeld genannt. 
thekstypologie auch als ,Studien- und Bildungsbibliothek' bezeichnet wurde, ${ }^{3}$ mit Blick auf die wissenschaftlich und kulturhistorisch relevanten Sammlungen ohne Weiteres als Wissenschaftliche Bibliothek fassen, der sich (auch) an ein wissenschaftlich mit diesen Materialien arbeitendes Publikum richtet. Während also ihre kulturhistorischen Sammlungen die ,bibliothèques patrimoniales‘, wie man sie mit einem klareren Begriff in der französischsprachigen Schweiz auch bezeichnet, zu den Wissenschaftlichen Bibliotheken rechnen lassen (um die vor allem in der Tradition der deutschsprachigen Länder vertraute Nomenklatur zu gebrauchen), eignet sich doch vorrangig die Zugehörigkeit zu bzw. der Versorgungsauftrag für eine Hochschule als massgebliches Kriterium, um zwischen reinen Kantonsbibliotheken und Kantonsbibliotheken, die zugleich einen Hochschulauftrag erfüllen, zu unterscheiden.

Auf die ausschliesslich mit kantonalem Mandat versehenen, meist kleineren Einrichtungen wird in der Folge nicht weiter eingegangen. ${ }^{4}$ Alle Bibliotheken mit kantonsbibliothekarischem Auftrag und die Schweizerische Nationalbibliothek arbeiten seit 2010 in loser Form in der Schweizerischen Konferenz der Kantonsbibliotheken (SKKB/CSBC) $)^{5}$ zusammen, die als Interessengruppe unter dem Dach des Berufsverbands Bibliothek Information Schweiz (BIS) eingerichtet wurde und als Verein organisiert ist. Eine Zuordnung zur politischen Ebene besteht formal nicht; Ansprechpartner für gemeinsame Initiativen ist naturgemäss aber die Konferenz der kantonalen Erziehungsdirektoren (EDK/CDIP, vergleichbar der Kultusministerkonferenz in Deutschland) in ihrer Zuständigkeit für die interkantonale Koordination in Kulturfragen. Die SKKB verfügt nicht über ein eigenes Programmbudget, so dass Initiativen für gemeinsame Dienstleistungen oder eine weitergehende Integration nur schwierig zu realisieren sind. ${ }^{6}$ Ein erstes Praxisbeispiel ist das Projekt Webarchiv Schweiz, in dem die Kantonsbibliotheken unter Koordination der Nationalbibliothek, die im Rahmen ihres Sammelauftrags für e-Helvetica auch die technische Plattform bereitstellt, landeskundlich wichti-

3 Siehe Robert Barth: Artikel ,Bibliotheken', Teil 2: Bibliothekstypen, Trägerschaft und Berufsverband. In: Historisches Lexikon der Schweiz. Stand des Artikels 2. Juli 2014 (www.hls-dhs-dss. ch/textes/d/D11299.php). Alle Links in diesem Beitrag wurden am 26.03.2018 überprüft.

4 Mir ist keine jüngere Überblicksdarstellung als die bei Christine Senser: Die Bibliotheken der Schweiz. Elemente des Buch- und Bibliothekswesens 13. Wiesbaden 1991, S. 102-120, bekannt.

5 Siehe www.skkb-csbc.ch sowie https://www.nb.admin.ch/snl/de/home/nb-professionell/pro jekte-und-programme/nationale-und-internationale-kooperation/skkb.html.

6 Insoweit haben sich die Hoffnungen, die aus den Darlegungen von Cornel Dora (Eine Bibliotheksstrategie für die Schweiz? In: Bibliothek - Forschung und Praxis 36 [2012], S. 78-86) sprechen, im Hinblick auf eine systemstärkende Rolle der SKKB und eine übergreifende bibliothekspolitische Steuerungsfunktion der Erziehungsdirektorenkonferenz bisher kaum erfüllt. 
ge Webseiten erschliessen und diese langfristig archivieren wollen. ${ }^{7}$ Eine mögliche Rolle könnte auch die Bündelung einer nationalen Initiative zur Bestandserhaltung sein, wie sie in Deutschland lanciert wurde ${ }^{8}$ - ein solches Anliegen wäre in gemeinsamer Verantwortung von Kantonen und Bund bei der EDK und beim Bundesamt für Kultur, bei dem auch die Schweizerische Nationalbibliothek angesiedelt ist, ${ }^{9}$ an der richtigen Stelle platziert.

\section{Bibliotheken im Hochschulbereich - eine Vielfalt organisatorischer Lösungen}

Mit der 2015 durch das Hochschulförderungs- und -koordinationsgesetz $(\mathrm{HFKG})^{10}$ vorgenommenen Neuordnung des schweizerischen tertiären Bildungswesens gliedert sich der Hochschulbereich in die drei Segmente der Universitäten einschliesslich der beiden Eidgenössischen Technischen Hochschulen, der durch das Fachhochschulgesetz ${ }^{11}$ von 1995 geschaffenen sieben Fachhochschulen ${ }^{12}$ und der Pädagogischen Hochschulen. ${ }^{13}$ Die Landschaft der Hochschulbibliotheken kann grundsätzlich entlang dieser Struktur nachgezeichnet werden. Im Segment der Universitäten können die reinen Universitätsbibliotheken, die, in ihrem Auftrag

7 Siehe www.nb.admin.ch/snl/de/home/nb-professionell/e-helvetica.html sowie https://www. nb.admin.ch/snl/de/home/nb-professionell/e-helvetica/infos-fuer-anbieter-innen/websites-_-we barchiv-schweiz.html.

8 Zur Organisation des Programms wurde an der Stiftung Preußischer Kulturbesitz die Koordinationsstelle zur Erhaltung des schriftlichen Kulturguts geschaffen (www.kek-spk.de).

9 Zur Schweizerischen Nationalbibliothek unten S. $38 \mathrm{ff}$.

10 Bundesgesetz über die Förderung der Hochschulen und die Koordination im schweizerischen Hochschulbereich (Systematische Sammlung des Bundesrechts [SR] 414.20, www.admin.ch/opc/ de/classified-compilation/20070429/index.html).

11 SR 414.71 (www.admin.ch/opc/de/classified-compilation/19950279).

12 Diese sind übergeordnete rechtlich-organisatorische Einheiten, die teilweise über Kantonsgrenzen hinweg reichen und durchweg mehrere Standorte, teils sogar mehrere eigenständige Institutionen aufweisen: die Haute Ecole Spécialisée de Suisse occidentale (HES-SO), die Berner Fachhochschule (BFH), die Fachhochschule Nordwestschweiz (FHNW), die Zürcher Fachhochschule (ZFH), die Fachhochschule Ostschweiz (FHO), die Scuola professionale universitaria della Svizzera italiana (SUPSI) und die Hochschule Luzern (HSLU). Der Grad der organisatorischen Selbständigkeit der Teileinrichtungen dieser Fachhochschulen variiert je nach gewählter rechtlicher Konstruktion.

13 In einigen Kantonen sind die Pädagogischen Hochschulen rechtlich-organisatorisch der jeweiligen Fachhochschule eingeordnet (so in Zürich und in der Nordwestschweiz). 
auf die Versorgung ihrer Hochschule und die Unterstützung von deren Forschung und Lehre fokussiert, zugleich auch organisatorisch ein genuiner Teil der Hochschule sind, von den Institutionen unterschieden werden, die neben der universitätsbezogenen Aufgabe eine öffentliche Zuständigkeit für Stadt, Region oder Kanton ausüben. Dieser Doppelauftrag ist in der Schweiz gegenüber der reinen Hochschulbibliothek aus historischen Gründen die verbreitetere Form. Die Doppelausrichtung kann dabei heute durchaus in der Trägerschaft der Hochschule ausgestaltet sein - in diesen Fällen hat in der Aufgabenwahrnehmung die universitäre Rolle in der Praxis zumeist das stärkere Gewicht -, in einigen Fällen aber auch nach wie vor in einer gegenüber der Hochschule externalisierten Organisationsform. ${ }^{14}$

Konstruktionen mit gemischten Aufträgen finden sich an vielen der grossen Hochschulstandorte der Schweiz. In Basel führt die Universitätsbibliothek in ihrer offiziellen Bezeichnung programmatisch den Namen der Öffentlichen Bibliothek der Universität Basel und unterstreicht damit das traditionell bürgerlich-republikanische Selbstverständnis der Universität des Stadtkantons, wo Stadt- und Universitätsgeschichte eng aufeinander bezogen sind, wie sich auch in den historischen Sammlungen der Bibliothek zeigt, die die Geschichte der ältesten Schweizer Universität und ihrer Gelehrten widerspiegelt. Gemäss dieser öffentlichen Rolle liegen auch die typisch kantonsbibliothekarischen Aufgaben bei der Baseler Universitätsbibliothek.

In den alten Universitätsstädten der Flächenkantone ist zudem der Typus der Stadt- und Universitätsbibliothek verbreitet oder doch bis in die jüngere Vergangenheit verbreitet gewesen. In Bern war bis zum Jahr 2007 die Stadt- und Universitätsbibliothek als gemeinsam von Stadt und Kanton getragene Stiftung die Zentralbibliothek der Universität, neben der eine grössere Zahl dezentraler Bibliotheken an der Universität selbst existierte. Parallel zu einer langfristig angelegten Standortentwicklungsplanung der Universität mit dem Ziel einer Konzentration auf eine begrenzte Zahl von Schwerpunktcampi kam es 2009 zur Integration der Stadt- und Universitätsbibliothek mit ihrem Standort in der historischen Berner Altstadt in die Strukturen der Universität. ${ }^{15}$ Dies kam auch im Namenswechsel der Gesamtinstitution zur „Universitätsbibliothek Bern“ zum Ausdruck, die nun alle bibliothekarischen Einrichtungen der Universität umfasst und mit der Zusammenführung des Personals sowie eines Teils der Erwerbungs-

14 Dazu siehe auch Noëmi Eglin-Chappuis: Governance von Universitätsbibliotheken: Optimierungsmöglichkeiten aus institutioneller Sicht. Chavannes-Lausanne 2009 (Cahier de l'IDHEAP 245), S. 19-24.

15 Siehe dazu die Jahresberichte der Bibliothek aus den Jahren 2007 bis 2009, verfügbar unter http://www.unibe.ch/universitaet/dienstleistungen/universitaetsbibliothek/ueber_uns/publikat ionen/index_ger.html. 
kredite einherging. Die frühere Zentralbibliothek im ältesten öffentlichen Bibliotheksgebäude der Schweiz hat den Namen „Bibliothek Münstergasse der UB Bern" und eine Funktion als fachübergreifende Studienbibliothek und Veranstaltungsort erhalten; hier werden aber unverändert auch die Bernensia gesammelt und präsentiert - die Wahrnehmung der kantonsbibliothekarischen Aufgaben ist also ungeachtet des organisatorischen Übergangs in die Universität an der Institution verblieben. ${ }^{16}$

Komplex ist die Situation in Zürich, dem grössten Hochschulstandort der Schweiz. In Anbetracht des Anlasses dieser Festschrift mag es erlaubt sein, hierauf etwas ausführlicher einzugehen. In Zürich findet man heute noch den Typus der zentralen Universitätsbibliothek, die organisatorisch ausserhalb der Strukturen der Universität steht. Die Zentralbibliothek Zürich war 1917 eine programmatisch verstandene Gründung, initiiert und politisch durchgesetzt von einem visionären Zürcher Bibliothekar, dem vormaligen Direktor der Stadtbibliothek Hermann Escher. Er strebte die Vereinigung von Beständen und Katalogen der auf das frühe 17. Jahrhundert zurückgehenden Stadtbibliothek, der seit 1835 gegründeten Kantonsbibliothek (die ihrerseits ältere Bestände, insbesondere aus dem mittelalterlichen Chorherrenstift, aufgenommen hatte) sowie einiger kleinerer gelehrter Büchersammlungen der Universität und der Zürcher Gymnasien an, um sie in einer Stiftung von Stadt und Kanton zusammenzuführen. So erhielt die wachsende Zürcher Universität eine leistungsfähige zentrale Bibliothek. Die Gründungsabsicht lässt sich bis heute am Namenszusatz „Kantons-, Stadt- und Universitätsbibliothek“ ablesen, der die Zweckbestimmung der neu geschaffenen Einrichtung aus dem Stiftungsvertrag wiedergibt. ${ }^{17}$ Die unverändert von beiden

16 Daneben pflegt die Bibliothek der Burgergemeinde Bern als Forschungsbibliothek und wissenschaftliches Archiv eine bedeutende Bernensiasammlung aus Handschriften, Nachlässen sowie Privat-, Firmen- und Gesellschaftsarchiven. Eine Graphische Sammlung und ein Photoarchiv kommen hinzu. Diese Verteilung der kulturhistorischen Sammlungen auf zwei Bibliotheken ist ein Sonderfall. Die Burgerbibliothek war 1951 aus der Stadtbibliothek herausgelöst worden, als die Stiftung Stadt- und Universitätsbibliothek Bern geschaffen wurde. Sie befindet sich in räumlicher Einheit mit der Bibliothek Münstergasse der Universitätsbibliothek. Die Burgergemeinde trägt auch das Zentrum Historische Bestände an der Bibliothek Münstergasse der Universitätsbibliothek.

17 Vertrag zwischen dem Kanton Zürich und der Stadt Zürich betreffend die Errichtung einer Zentralbibliothek als öffentliche Stiftung (Stiftungsvertrag), online verfügbar in der Zürcher Gesetzessammlung (ZH-Lex) 432.21 (www.zh.ch/internet/de/rechtliche_grundlagen/gesetze/erlass. html?Open\&Ordnr=432.21). Der Stiftungsvertrag wurde 1910 zwischen Stadt und Kanton geschlossen, 1914 wurde der Neubau vom Volk gutgeheissen und 1917 schliesslich nahm die Zentralbibliothek ihren Betrieb im neuen Gebäude am Zähringerplatz in der Zürcher Altstadt auf. Siehe die Darstellung bei Rea Brändle u.a.: Wissen im Zentrum. 100 Jahre Zentralbibliothek Zürich. Zürich 2017, bes. S. 20-33 und 237-241. 
Ständen getragene öffentliche Stiftung ${ }^{18}$ ist in ihrer Organisation dem im Gründungsvertrag von 1910 gewählten Konstrukt treu geblieben, der zusammen mit dem Bauprojekt in der Zürcher Altstadt 1914 in beiden Volksabstimmungen in der Stadt und im Kanton Zürich breite Mehrheiten gefunden hatte. Im Zuge der Bibliotheksentwicklung an der Universität hat sich in der zweiten Hälfte des 20. Jahrhunderts an der Zentralbibliothek ein fachlicher Schwerpunkt in den buchorientierten Fächern der Geistes- und Kulturwissenschaften ausgeprägt, der in der aktuellen Entwicklungsstrategie ${ }^{19}$ mit der Ausweitung auf den E-Book-Bereich akzentuiert und zugleich unterstrichen wird - eine typische Ausprägung gerade beim Typus der wissenschaftlichen Allgemeinbibliotheken der Schweiz, die zusätzlich eine universitäre Funktion wahrnehmen.

Zugleich hat sich im selben Zeitraum das Bibliothekswesen an der Universität selbst, wie an vielen traditionellen Universitäten, stark entwickelt und diversifiziert. $^{20}$ Es wuchs - insbesondere in den Fächern der Philosophischen Fakultät die Zahl teils kleiner, teils mittlerer Instituts- und Fakultätsbibliotheken, die weitgehend von der Zentralbibliothek unabhängig agierten und stark auf die Bedürfnisse der Institute und ihrer Lehrstühle ausgerichtet wurden. Anders verlief die Entwicklung in den Naturwissenschaften: In die Planung eines neuen Campus ausserhalb des Stadtzentrums seit den 1960er Jahren wurde von Anfang an - der allgemeinen Tendenz an neugegründeten Hochschulbibliotheken dieser Zeit folgend - eine zentrale Bereichsbibliothek einbezogen. Schliesslich 1980 eröffnet, erhielt sie den Namen „Hauptbibliothek der Universität Zürich“ und begann mit der Zeit über die Literaturversorgung der naturwissenschaftlichen Fächer auf dem Campus Irchel hinaus gewisse zentrale Aufgaben für die Universität wahrzunehmen, so im Bereich der E-Medien-Lizenzierung oder in jüngerer Zeit durch den Aufbau eines institutionellen Open Access-Repository. Einen koordinierenden Einfluss auf das Gesamtsystem der Bibliotheken an der Universität hat sie aber nur begrenzt entfalten können.

18 Nachdem das Budget der Zentralbibliothek zunächst je zur Hälfte von Stadt und Kanton getragen worden war, wurde im Jahr 1985 dem gewichtiger gewordenen Anteil der ZB an der Literaturversorgung der Universität dadurch Rechnung getragen, dass nunmehr vier Fünftel der Finanzierung vom Kanton und ein Fünftel von der Stadt geleistet werden (vgl. Mario König: Ein Kampf ums Geld. Die Bibliothek und ihre Stifter. In: Brändle u.a., Wissen [wie Anm. 17], S. 232271, hier S. 261-263). Dieser Finanzierungsschlüssel wird weiterhin angewendet.

19 Strategie ZB 2020. Die Zentralbibliothek Zürich zeigt Profil, 2016, S. 9 und 12 (www.zb.uzh.ch/ Medien/strategiebroschuere_161117.pdf).

20 Dazu Wilfried Lochbühler: Dreischichtigkeit zwischen Diversifikation und Kooperation. In: Konstanze Söllner, Wilfried Sühl-Stromenger (Hrsg.): Handbuch der Hochschulbibliothekssysteme: Leistungsfähige Informationsinfrastrukturen für Wissenschaft und Studium. Berlin/Boston 2014, S. 112-120. 
Man kann für die Universität Zürich also in gewissem Sinne von einem dreischichtigen Versorgungssystem sprechen $^{21}$, und es überrascht kaum, dass immer wieder Bemühungen um eine bessere Abstimmung und Steuerung dieser verschiedenen, nicht durch eine durchgehende gemeinsame Governance verbundenen Ebenen lanciert wurden. Aktuell hat in der Universität ein von der Hochschulleitung initiierter, bis Mitte der 2020er Jahre angelegter Reorganisationsprozess begonnen, der eine einheitliche Organisations- und Dienstleistungsstruktur für die Bibliotheken in der direkten Zuständigkeit der Universität implementieren und insbesondere die hochdiversifizierte Situation in der Philosophischen Fakultät konsolidieren will. Wie schon bei der Planung des Campus Irchel, wird die organisatorische Massnahme von Immobilienplanungen der Universität flankiert und steht im Kontext der städtebaulichen Entwicklung des Hochschulquartiers im Zürcher Stadtzentrum, die bis zum Jahr 2025 auch ein neues Kollegiengebäude mit einer gemeinsamen Bibliothekseinheit bringen sollen.

Man kann freilich die Bibliothekssituation am Hochschulstandort Zürich nicht behandeln, ohne die andere grosse Wissenschaftliche Bibliothek am Ort zu erwähnen, die zugleich eine nationale Funktion erfüllt und vermutlich unter den Schweizer Bibliotheken diejenige ist, die am stärksten auch internationale Ausstrahlung gewonnen hat, nicht zuletzt durch ihre Vorreiterposition unter den Schweizer Bibliotheken bei der digitalen Transformation des Informationsmanagements. ${ }^{22}$ Die Bibliothek der Eidgenössischen Technischen Hochschule (ETH) ist integraler Bestandteil ihrer Hochschule und wie der gesamte ETH-Bereich, zu dem auch noch die Ecole Polytechnique Fédérale in Lausanne und eine Reihe von Forschungsanstalten gehören, allein durch den Bund finanziert. Die ETH-Biblio-

21 Der Begriff ist allerdings missverständlich, weil es sich - bisher jedenfalls - nicht um ein abgestimmtes System dreier Ebenen mit zueinander komplementären Leistungsaufträgen und Subsidiaritäten handelt, sondern zumindest teilweise um das Ergebnis paralleler, unabhängig nebeneinander verlaufener Entwicklungen der letzten Jahrzehnte. Organisatorisch bestehen tatsächlich mit der Stiftung Zentralbibliothek, der für die Mathematisch-Naturwissenschaftliche und die Medizinische Fakultät zuständigen Hauptbibliothek und den bisher faktisch autonomen Instituts- und Fakultätsbibliotheken der Universität drei selbständige Sphären, in denen die Personalstellen, die Verfügung über die finanziellen Ressourcen und die Entscheidungsstrukturen bislang nicht koordiniert wurden.

22 Überblicksdarstellungen finden sich in den Beiträgen von Wolfram Neubauer: Die Informationsinfrastrukturen an der ETH Zürich. In: Zeitschrift für Bibliothekswesen und Bibliographie 61 (2014), S. 224-230, sowie Arlette Piguet: Die ETH-Bibliothek: eine unentbehrliche Dienstleisterin für Forschung und Lehre. In: Rafael Ball, Stefan Wiederkehr (Hrsg.): Vernetztes Wissen. Online. Die Bibliothek als Managementaufgabe. Festschrift für Wolfram Neubauer zum 65. Geburtstag. Berlin/Boston 2015, S. 3-15. 
thek ist so in erster Linie der Unterstützung von Studium, Lehre und Forschung an einer Einrichtung verpflichtet, die - zusammen mit der jüngeren Schwesterhochschule in Lausanne (und ihrer freilich deutlich kleineren Bibliothek, die Teil des architektonisch spektakulären Rolex Learning Center ist) - als einzige Hochschule der Schweiz seit Jahren vordere Plätze in den internationalen Universitätsrankings besetzt. Über die Versorgung der ETH hinaus nimmt sie aber auch die Funktion eines nationalen Informations- und Kompetenzzentrums für Technik und Naturwissenschaften wahr und ist insofern - ein Sonderfall unter den Schweizer Bibliotheken - mit den zentralen Fachbibliotheken der Leibniz-Gemeinschaft in Deutschland zu vergleichen. Neben dem Betrieb des NEBIS-Verbunds ist unter den Dienstleistungen mit überregionaler oder nationaler Reichweite auch der DOI-Desk zu erwähnen, der die offizielle DOI-Registrierungsstelle für den Schweizer Hochschul- und Forschungssektor zur Vergabe von Digital Object Identifiern für wissenschaftliche Publikationen und Forschungsdatensets ist. Zu diesem Zweck ist die ETH Zürich Mitglied bei der internationalen Organisation DataCite ${ }^{23}$, die bei der Technischen Informationsbibliothek in Hannover angesiedelt ist.

In Genf - um an das andere Ende der Schweiz zu wechseln - wurden die Bibliotheken der Universität, konzentriert an vier Hauptstandorten, erst 2008 organisatorisch unter die Leitung einer zentralen „Division de l'information scientifique“ (DIS) gestellt, während sie zuvor den einzelnen Fakultäten und Departementen der Universität zugeordnet waren. Die DIS ist also keine klassische zentrale Universitätsbibliothek, sie nimmt vielmehr Steuerungs- und Koordinationsaufgaben innerhalb eines Gesamtsystems „Bibliothèque de l'Université de Genève“ wahr. ${ }^{24}$ Die Koordinationsleistungen beziehen sich vor allem auf interne bibliothekarische Geschäftsprozesse, das Webangebot mit Rechercheund Zugriffstools und das institutionelle Dokumentenrepository. Wie auch an anderen Hochschulen mit traditionell schwach ausgeprägter Zentralisierung des Bibliothekssystems liegt ein Akzent auf der lokalen Verbundkoordination, hier innerhalb des Westschweizer Bibliotheksverbunds, ${ }^{25}$ und auf der Verwaltung der elektronischen Ressourcen. Mit Blick auf die Standortkonsolidierung hat man eine gewisse Bündelung von Fachbereichsbibliotheken an räumlichen Kristallisationskernen der über die Stadt Genf verteilten Universität erreicht, so dass heute der grösste Teil des bibliothekarischen Geschehens auf vier ,sites` konzentriert ist;

23 Das derzeit einzige weitere Mitglied aus der Schweiz ist das - selbst internationale - Forschungszentrum CERN in Genf-Meyrin.

24 Siehe www.unige.ch/dis/presentation.

25 Dazu siehe Anm. 33. 
eine eigentliche Universitätsbibliothek als zentraler Benutzungs- und Serviceort und infrastruktureller Kern ist dabei aber nicht geschaffen worden. Die zentrale wissenschaftliche Bibliothek in Genf ist demgegenüber seit jeher die weiterhin in städtischer Trägerschaft stehende Bibliothèque de Genève. Sie war historisch die zentrale Bibliothek der Universität und trug darum bis zur organisatorischen Trennung im Jahr 2006 auch den Namen „Bibliothèque publique et universitaire de Genève“. Sie verwahrt die kulturhistorischen Bestände und sammelt die Genfer Publikationen, übernimmt zugleich aber auch vertraglich verankerte Literaturversorgungsaufgaben einer Zentralbibliothek für die Universität in den geisteswissenschaftlichen und historischen Disziplinen ${ }^{26}$ - ganz ähnlich, wie es bei der Zürcher Zentralbibliothek festzuhalten war.

Ähnlich stellen sich die Verhältnisse auch im kleineren Neuenburg dar: Die Kantonsbibliothek erhielt schon zu Beginn des 20. Jahrhunderts die Rolle der Universitätsbibliothek der Universität - weiterhin lautet der Name „Bibliothèque publique et universitaire de Neuchâtel“ -, ist aber auch nach der Neuordnung im Jahr 1983, in der die gemeinsame Trägerschaft von Stadt und Kanton fixiert wurde, organisatorisch, darin vergleichbar der Zentralbibliothek Zürich, ausserhalb der Strukturen der Universität geblieben. Die Bibliotheken der Universität wiederum besitzen eine auch formal ausgeprägte Koordination mit einer eigenständigen Leitungsebene, gemeinsamen Serviceangeboten (darunter auch hier der Betrieb eines Subverbundes innerhalb des Westschweizer Bibliotheksverbunds für die Kantone Neuenburg und Jura) und einer einheitlichen Aussenrepräsentation. Eine eigene, institutionell in die Universität eingegliederte Zentralbibliothek gibt es nicht.

Noch in zwei weiteren Kantonen findet sich der Typus der integrierten Kantons- und Universitätsbibliothek, den wir in Basel, Bern, Zürich und Neuenburg angetroffen haben: so im Kanton Waadt mit der Bibliothèque cantonale et universitaire de Lausanne und im zweisprachigen Kanton Freiburg mit der Bibliothèque cantonale et universitaire de Fribourg. In beiden Fällen handelt es sich um Organisationen, die von der jeweiligen kantonalen Verwaltung abhängen. Während in Lausanne die BCU neben der Rolle als Kantonsbibliothek auch das - an einer überschaubaren Zahl von ,sites‘ konzentrierte - Bibliothekswesen der Universität in ihrer alleinigen Zuständigkeit führt, ist dies in Freiburg zumindest formell weniger eindeutig: Die BCU ist dort direkter Teil der staatlichen Verwaltung und koordiniert das Bibliothekswesen der Universität Freiburg in fachlicher

26 Convention entre l'Université de Genève et la Ville de Genève fixant les modalités de collaboration de leurs bibliothèques (www.unige.ch/dis/files/7414/1215/7642/Convention_UNIGE_ VdG_02022010.pdf). 
Hinsicht ${ }^{27}$, wobei die zahlreichen dezentralen Bibliotheken selbst aber organisatorisch zur Universität und ihren Fakultäten gehören. In der Praxis ist die Integration aber weit fortgeschritten. Beide Einrichtungen haben als Kantonsbibliotheken explizit auch einen Koordinationsauftrag für die übrigen vom Kanton getragenen kleineren Bibliotheken und sorgen u. a. für deren Verbundanschluss.

Die Freiburger Konstruktion hat gewisse Parallelen im Kanton Luzern, wo die Zentral- und Hochschulbibliothek (ZHB) Luzern die zentrale wissenschaftliche Informationseinrichtung ist. Auch sie ist eine direkt der kantonalen Verwaltung eingegliederte Institution und war seit ihrer Gründung 1951 - einmal mehr eine Fusion aus einer städtischen Bürgerbibliothek und der im 19. Jahrhundert eingerichteten Kantonsbibliothek - mit dem Auftrag der Bewahrung, Ergänzung und Vermittlung der Lucernensia ausgestattet. Luzern hat erst 1970 eine Universität erhalten, die aus der zuvor selbständigen, mit ihren Vorgängerinstitutionen bis in die Zeit der Gegenreformation zurückreichenden Katholisch-Theologischen Fakultät hervorgegangen ist. Heute bilden vier Fakultäten die weiterhin eher kleine, geistes- und gesellschaftswissenschaftlich ausgerichtete Universität, im Übrigen die einzige der Zentralschweiz. Die ZHB Luzern organisiert und betreibt in überschaubarer Entfernung zum Stammhaus die Bibliothek der Universität im zentralen Lehr- und Verwaltungsgebäude sowie an weiteren Standorten (teilweise sogar ausserhalb von Luzern) die Bibliotheken der Luzerner Fachhochschule und der interkantonalen Pädagogischen Hochschule der Zentralschweiz in sogenannter ,Auftragsverwaltung ' und erhält dafür von den Hochschulen gesondert zur Verfügung gestellte Budgets.

Unter den universitären Bibliotheken sind demgegenüber erstaunlich wenige Einrichtungen als reine Hochschulbibliotheken mit exklusivem Versorgungsauftrag für die Hochschule organisiert. Diese Konstellation wurde dort begünstigt, wo es einerseits verhältnismässig spät zur eigentlichen Universitätsgründung

27 „Die Departements-, Fakultäts- und interfakultären Bibliotheken der Universität Freiburg sind in bibliothekarischer Hinsicht Teil der KUB“, heisst es in der Selbstdarstellung (www2.fr.ch/bcuf/ Dynamic.aspx?c=2543). Entsprechend legt das Reglement der Bibliothek (http://www2.fr.ch/ bcuf/Dynamic.aspx?c=34) fest: „Der Direktor [...] sorgt dafür, dass in der Zentralbibliothek und in den dezentralen Bibliotheken an der Universität dieselben bibliothekarischen Grundsätze angewandt werden“ (Art. 1 Abs. 1 lit.b). Das Reglement regelt diese Zusammenarbeit ausführlich und detailliert: So soll etwa „für jeden Bereich [...] ein Konzept für die Aufteilung der Werke auf die Zentralbibliothek und die dezentralen Bibliotheken [erstellt werden]. Jedes Aufteilungskonzept muss vom Direktor und vom Rektorat der Universität genehmigt werden“ (Art. 8 Abs. 1-2). Zentralbibliothek und die Bibliotheken der Universität verfügen über getrennte Erwerbungsbudgets, aber die Anschaffungen sind abzustimmen (Art. 8 Abs. 4 und Art. 11), und auch das Personal wird separat angestellt, wobei der Direktor der Zentralbibliothek bei Anstellungen für die universitären Bibliotheken angehört werden muss (Art. 9 Abs. 3). 
kam und andererseits am Ort bereits, davon unabhängig, eine öffentliche wissenschaftliche Allgemeinbibliothek etabliert war. Die Universitätsbibliothek der Universität St. Gallen ist die Bibliothek der, historisch gesehen, noch recht jungen „Hochschule St. Gallen“ (HSG) - so der Name bis zum Jahr 1993, unter dem die 1963 in der heutigen Form entstandene Institution mit dem Schwerpunkt in Management- und Wirtschaftswissenschaften bekanntgeworden ist. Die Rolle der Kantonsbibliothek übt demgegenüber die in die Reformationszeit zurückgehende Vadiana aus, während die zum Katholischen Konfessionsteil des Kantons St. Gallen (öffentlich-rechtliche Körperschaft) gehörende Stiftsbibliothek ihre international bedeutenden Altbestände in den spätbarocken Räumen des zum UNESCO-Weltkulturerbe gehörenden St. Galler Klosterbezirks aufbewahrt und präsentiert.

Schliesslich liefert die italienischsprachige Schweiz mit der Bibliothek der Università della Svizzera italiana ein Beispiel für eine junge Hochschulgründung, die 1996 zu einer eigenen Universitätsbibliothek auf dem zentralen Campus in Lugano geführt hat. Sie ist von der bereits etablierten Kantonsbibliothek unabhängig geblieben. ${ }^{28}$ Dabei weist die Tessiner Situation die Besonderheit auf, dass die Kantonsbibliothek - die nicht nur in Lugano, sondern auch im Kantonshauptort Bellinzona und in Mendrisio präsent ist-überdies den regionalen Bibliotheksverbund und das Bibliotheksverwaltungssystem betreibt, in dem auch die Universitätsbibliothek arbeitet, eine Konstellation, die sich aktuell unter den Vorzeichen der Neuordnung der Schweizer Verbundlandschaft, von der noch die Rede sein wird, aber ändern wird.

\section{Eine föderalistisch geprägte Landschaft und die Rolle der Nationalbibliothek}

Der gegebene Überblick über die Situation in den Kantonen und an den Hochschulstandorten zeigt bereits den stark föderal geprägten Charakter der Schweizerischen Kultur-, Wissenschafts- und Bildungslandschaft, in der aufgrund der Kulturhoheit der Kantone der Bund - mit Ausnahme der (freilich Hochschulautonomie geniessenden) beiden Eidgenössischen Technischen Hochschulen und einiger angegliederter Forschungsinstitutionen - traditionell kaum eine Rolle innehatte. Ansätze zu einer ernsthaften Koordination auf nationaler Ebene und zu

28 it.bul.sbu.usi.ch. Neben dem Hauptstandort Lugano gibt es einen weiteren an der Architekturfakultät in Mendrisio. 
einem Aufbau zentral organisierter und bereitgestellter Services für die gesamte wissenschaftliche Bibliothekslandschaft sind erst seit den 2000er Jahren festzustellen, angestossen durch die finanzielle Förderung des Bundes zur Sicherung der Wettbewerbsfähigkeit der Hochschulen im Umfeld von Internationalisierung und Digitalisierung, die auch auf den Bereich der wissenschaftlichen Informationsinfrastrukturen zielt. ${ }^{29}$

Ein weiterer Ausdruck dieser föderal geprägten Kultur- und Bildungslandschaft ist die Stellung der Nationalbibliothek, die bisher für das Gesamtsystem nur in einzelnen Bereichen eine strukturbildend-koordinierende Rolle spielt. ${ }^{30}$ In dieses Bild fügt sich, dass sie als Teil des (seinerseits noch historisch jungen) Bundesamts für Kultur eine unselbständige Einrichtung innerhalb des eidgenössischen Departements des Inneren ist. Wie in anderen kulturföderalistisch orientierten Ländern auch, ist die Schweizerische Nationalbibliothek, im deutschen Sprachraum der Schweiz bis 2006 mit dem Namen „Schweizerische Landesbibliothek“, eine relativ junge Gründung (1895), seit 1931 in einem ikonischen Bibliotheksbau der Neuen Sachlichkeit in Bern untergebracht und in ihrer Sammlungsund Vermittlungstätigkeit ausschliesslich auf Helvetica ausgerichtet. Dass der

29 Mit sog. projektgebundenen Beiträgen des Bundes wurden über die Schweizerische Universitätskonferenz (SUK) das Projektcluster e-lib.ch: Elektronische Bibliothek Schweiz (Laufzeit 2008-2012) und das Förderprogramm „Wissenschaftliche Information: Zugang, Verarbeitung und Speicherung“ (SUK P-2, Laufzeit 2013-2016) lanciert, seit 2017 mit neuer Bezeichnung P-5 (www. swissuniversities.ch/de/organisation/projekte-und-programme/p-5). Der förderpolitische Rahmen für das Programm SUK P-2/P-5 wurde in einem Strategiepapier (Bündelung der Kräfte in der wissenschaftlichen Information: nationale Strategie. Hrsg. vom Programm P-2 „Wissenschaftliche Information: Zugang, Verarbeitung und Speicherung“ der Schweizerischen Universitätskonferenz 2014) und in einer darauf aufbauenden Umsetzungsvision (White Paper for a Swiss Information Provisioning and Processing Infrastructure 2020. Hrsg. vom Programm P-2 „Wissenschaftliche Information: Zugang, Verarbeitung und Speicherung“ der Schweizerischen Universitätskonferenz 2014, ergänzt 2015) gezogen. Vgl. dazu die Beiträge „Nationale Förderprogramme“ von Alice Keller und „Das Programm P-5“ von Gabi Schneider in diesem Band. Die aktuell geförderten Projekte leiten sich aus der Umsetzungsstrategie 2017-2020 ab (www.swissuniversities.ch/fileadmin/swiss universities/Dokumente/Organisation/SUK-P/SUK_P-2/Umsetzungsstrategie_2017-2020_DE.pdf).

30 Dafür bestünde freilich im Auftrag der Nationalbibliothek Spielraum, heisst es doch in Art. 2 des Gesetzes über die Nationalbibliothek (SR 432.21): „Sie trägt zur Entwicklung des nationalen und internationalen Bibliothekswesens bei“ und in Art. 10: „Sie stellt in enger Zusammenarbeit mit andern grossen öffentlichen Bibliotheken die Koordination sicher, namentlich im Bereich der Bibliotheksautomatisierung." Die Nationalbibliothek verfügt auch über eine vom Bundesrat berufene Kommission, die beratend in übergeordneten Fragen des Schweizerischen Bibliothekswesens wirken soll: Sie „bearbeitet Fragen der gesamtschweizerischen Politik auf dem Gebiet der wissenschaftlichen Information und Dokumentation unter Berücksichtigung der allgemeinen Wissenschaftspolitik“ (https://www.nb.admin.ch/snl/de/home/die-schweizerische-nationalbibl iothek--alles-ueber-die-schweiz/organisation/kommission-der-nationalbibliothek.html). 
eigene Schweizer Publikationsmarkt angesichts der kulturellen Nähe zu den grossen Nachbarländern naturgemäss von begrenzter Grösse ist, wirkt sich auch auf die Dimension der Tätigkeit der Nationalbibliothek aus. Sie nimmt die nationalbibliographische Verzeichnung und die Sammlung der Belegexemplare der Schweizerischen Buchproduktion sowie der Literatur über die Schweiz wahr, stellt sie vor Ort in Bern dem Publikum zur Verfügung und betreibt ein Informationszentrum zur Schweiz. Seit 2011 sammelt sie auch e-Helvetica und trägt die Verantwortung für deren Langzeitarchivierung. Schliesslich vertritt die Nationalbibliothek die Schweiz in internationalen Standardisierungsgremien. Seit 1991 übt sie mit dem Schweizerischen Literaturarchiv auch archivische Aufgaben aus und ist dazu internationale Kooperationen eingegangen. Seit 2016 gehört zudem die Schweizerische Nationalphonothek in Lugano zur Bibliothek. In der Konferenz der europäischen Nationalbibliotheken und in multinationalen Projekten, die - wie Europeana und The European Library - der Digitalisierung und Vermittlung des kulturellen Erbes der europäischen Länder gewidmet sind, repräsentiert sie die Schweiz.

\section{Verbundstrukturen im Umbruch}

Die bibliothekarische Kooperation der Wissenschaftlichen Bibliotheken der Schweiz hat sich bisher vor allem in den einerseits regional, andererseits an den Sprachgrenzen orientierten Bibliotheksverbünden institutionalisiert. Wie andernorts, ist ihre Entstehung auch hierzulande mit dem Möglichwerden des standortübergreifenden Betriebs von (integrierten) Bibliotheksverwaltungssystemen über Datennetze in den 1980/1990er Jahren verbunden. Die Metamorphosen der eingesetzten technischen Systeme und die organisatorischen Konsequenzen der diversen Ablösungen können an dieser Stelle nicht im Einzelnen nachgezeichnet werden. ${ }^{31}$ Die Wissenschaftlichen Bibliotheken der Schweiz sind in ihrer grossen Mehrheit heute in zwei Verbundwelten aufgeteilt, die des Informationsverbundes

31 Dazu siehe Robert Barth, Gabi Schneider: „Die Zukunft hat noch nicht begonnen“: die Automatisierung der Hochschulbibliotheken in der Schweiz 1965 bis 1995. In: Für alle(s) offen. Bibliotheken auf neuen Wegen: Festschrift für Dr. Fredy Gröbli, Direktor der Öffentlichen Bibliothek der Universität Basel. Basel 1995, S. 26-37 sowie mit einem knappen Überblick Dora, Bibliotheksstrategie (wie Anm. 6), hier S. 81f. Zuletzt Tobias Viegener: Die Schweizer Verbundlandschaft ein Hemmnis für die Entwicklung der Bibliotheken? In: 027.7 Zeitschrift für Bibliothekskultur 1 (2013), S. 74-80, hier S. 76f. (http://dx.doi.org/10.12685/027.7-1-2-29). Zur aktuellen Situation siehe auch die Ausführungen im Beitrag „,Gemeinsam sind wir stärker““ von Wolfram Neubauer in diesem Band. 
Deutschschweiz (IDS) mit vier Teilverbünden ${ }^{32}$, in denen seit 1998 das Bibliothekssystem Aleph500 des Anbieters Ex Libris in separaten Installationen betrieben wird, und die des Réseau des bibliothèques de Suisse occidentale oder kurz Réseau romand (RERO) mit sechs Subverbünden ${ }^{33}$, jedoch einer einzigen technischen Installation in der Verbundzentrale in Martigny im Kanton Wallis, nämlich des Systems Virtua des Anbieters Innovative Interfaces.

Aufgrund der betrieblichen Zentralisierung und des gemeinsamen Datenpools erscheint das Réseau romand als der stärker vereinheitlichte und tiefer integrierte Verbund; tatsächlich gilt dies z. B. auch unter dem Aspekt der Formatund Regelwerksanwendung. Demgegenüber ist der IDS eigentlich eine eher lockere Verbundkonföderation mit der zwar gleichen, aber jeweils unabhängig betriebenen systemtechnischen Grundlage. Harmonisierung gab es aber auch hier von Anfang an bei Regelwerk und Austauschformat sowie bei grundlegenden Benutzungsstandards wie einer gemeinsamen Benutzerverwaltung. ${ }^{34}$ Grundlage der Kooperation war jedoch die gemeinsame Beschaffung des Bibliothekssystems in der Mitte der 1990er Jahre. Unter den IDS-Verbünden gibt es erhebliche Grössenunterschiede zwischen dem von der ETH-Bibliothek betriebenen grössten Teilverbund NEBIS am einen Ende als einem schweizweit ausgreifenden Netzwerk, das freilich mit den grossen Partnern ETH-Bibliothek, Zentralbibliothek Zürich

32 NEBIS, IDS Basel/Bern, IDS Luzern und IDS St. Gallen. Dazu kommen die sogenannten ,IDSPartner ' in IDS-externen Bibliotheken und Verbünden sowohl in der Schweiz (kantonale Verbünde im Aargau, in St. Gallen, im Tessin und in Graubünden) als auch im benachbarten Ausland (Liechtenstein, Österreich, Luxemburg). Sie nutzen ebenfalls das Bibliothekssystem Aleph500 auf eigenen Installationen und haben Zugang zu bestimmten Dienstleistungen des IDS-Gesamtverbundes (Datenpool für Katalog-, Norm- und Fremddaten, Systemunterstützung, Schulungen).

33 Aufgrund des zentralen technischen Betriebs in der RERO-Verbundzentrale ist der Begriff Subverbünde womöglich nicht optimal. In RERO spricht man von „coordinations locales“, verteilten Servicezentralen, die den direkten Support für die Bibliotheken in ihrem Zuständigkeitsbereich leisten und in den Hochschulkantonen in der Regel an der jeweiligen Universitätsbibliothek angesiedelt sind: Kanton Freiburg, Kanton Genf, Kanton Wallis, Kantone Neuenburg/Jura sowie die Koordination für die Bibliotheken der in RERO organisierten Bundesinstitutionen (v.a. das Bundesgericht an seinen verschiedenen Standorten und das Bundesamt für Justiz; die Koordination ist angesiedelt am Schweizerischen Institut für Rechtsvergleichung in Lausanne).

34 Zum gemeinsamen Benutzerdatenpool, der vor allem mit Blick auf den Aufbau eines IDSweiten Kurierservice als Speziallösung mit einer eigenen Aleph-Datenbank und Replikationsverfahren zwischen den technisch selbständigen IDS-Subverbünden eingerichtet wurde, siehe Ulrich Niederer: The Shared User File of the University Libraries in German Speaking Switzerland as a Tool for Cooperation. In: LIBER Quarterly 16/1 (2006) (http://doi.org/10.18352/lq.7831). Im Projekt SLSP wird für die Zukunft ein Identitäts- und Rollenmanagement für die Benutzerdatenverwaltung angestrebt, das den Anschluss an das webbasierte Identitätsmanagement im Hochschulumfeld herstellt; dazu siehe unten S. 49f. 
und Universität Zürich sein Gravitationszentrum am Wissenschaftsplatz Zürich findet (eine Gewichtung, die auch in der Governancestruktur des Verbunds ihre Entsprechung besitzt), und den viel kleineren Verbünden in St. Gallen oder Luzern am anderen. Eine Mittelstellung hinsichtlich der Grösse nimmt der IDS Basel/Bern ein, bei dem sich die beiden Kantone und Universitäten Aufgaben und Finanzierung teilen.

Für übergreifende Fragen wurde im IDS eine institutionalisierte Verbundkoordination eingerichtet, die u.a. für das bis 2016 gültige Regelwerk KIDS (Katalogisierungsregeln des IDS, eine Adaptation der AACR2) verantwortlich war und seitdem die Anwendung des neu eingeführten Regelwerks Resource Description and Access (RDA) im deutschsprachigen Anwendungsprofil D-A-CH für alle IDS-Teilnehmer koordiniert. Die IDS-Verbundkoordination vertritt die Bibliotheken in dem bei der Deutschen Nationalbibliothek angesiedelten Standardisierungsausschuss sowie weiteren ihm angegliederten Expertengremien der D-A$\mathrm{CH}$-Kooperation. Unvermeidlich haben sich allerdings in den vier IDS-Subverbünden jenseits des gemeinsamen Regelwerks doch Praxisunterschiede in Formatund Anwendungsfragen und bei der (insbesondere inhaltlichen) Erschliessung herausgebildet, ${ }^{35}$ die nun im Zuge des Wechsel zu einer schweizweiten Katalogisierungs- und Datenplattform zu harmonisieren sind. Als Auftraggeber und Steuerungsinstanz des IDS-Supraverbunds und der Verbundkoordination fungiert die Konferenz der Deutschschweizer Hochschulbibliotheken, die 2003 den rechtlich eigenständigen Verein Informationsverbund Deutschschweiz gegründet hat. Folgerichtig wird die Aufsicht über den IDS und die Verbundkoordination von den Direktorinnen und Direktoren der beteiligten Bibliotheken wahrgenommen. Mit dem Übergang zu einer neuen Struktur im Projekt SLSP will sich der IDS samt Verein und Koordinationsstelle auflösen, die Mandate und Vertretungen sollen künftig von SLSP geführt werden.

Im Unterschied zum IDS, dessen Mitglieder die Hochschulen mit ihren Biblio-

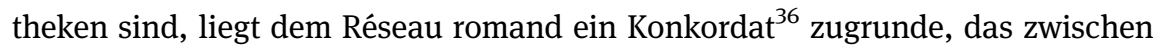
der Westschweizer Universitätskonferenz und den teilnehmenden Kantonen (seit 2015 ohne den Kanton Waadt), der Westschweizer Fachhochschule (Haute Ecole Spécialisée de Suisse occidentale, HES-SO) sowie dem Bundesgericht geschlossen wurde. Das Aufsichtsgremium ist seit 2009 die Westschweizer Erziehungsdirektorenkonferenz CIIP. Auch wenn die Westschweizer Universitäten und Hochschulen

35 Dazu auch Viegener, Schweizer Verbundlandschaft (wie Anm. 31), S. $77 \mathrm{f}$.

36 Convention relative à la gestion et à l'administration du Réseau des bibliothèques de Suisse occidentale, Fassung vom 01. Januar 2000 (www.rero.ch/pdfview.php?section=infos\&filename= Convention_RERO.pdf). Der Ursprung des Konkordats reicht ins Jahr 1985 zurück. 
naturgemäss die grossen Teilnehmer im Verbund sind und die Hochschulleitungen eine wesentliche Steuerungsfunktion ausüben, ergibt sich aus dieser Struktur auch ein erheblicher Einfluss der fünf Kantonsregierungen und -parlamente. Im Einklang damit bezieht der Finanzierungsschlüssel des Réseau romand neben der Zahl der Hochschulangehörigen im jeweiligen Kanton auch die Gesamteinwohnerzahl je Kanton ein.

Das in diesem Band noch ausführlich beschriebene, mit Fördermitteln der Eidgenossenschaft auf den Weg gebrachte Projekt SLSP zum Aufbau einer gemeinsamen Serviceplattform der Schweizer Hochschulbibliotheken ${ }^{37}$ mit einem zentralen, cloudbasierten Bibliotheksverwaltungssystem der neuen Generation als Kern soll bis zum Jahr 2020/2021 zur Auflösung des IDS-Netzwerks samt seiner Teilverbünde und $\mathrm{zu}$ einem gemeinsamen Systemangebot für alle Schweizer Hochschulbibliotheken unabhängig von Regionen und Sprachgrenzen führen. Diese Initiative wurde dadurch beschleunigt, dass der Kanton Waadt im Jahr 2014 das Réseau romand verlassen und, ebenfalls auf der Basis eines Next GenerationSystems (Ex Libris Alma), einen eigenen kantonalen Bibliotheksverbund aufgebaut hat. Seit 2016 ist dieser Verbund - mit der wortspielerischen Namensschöpfung „Renouvaud“ benannt - in Betrieb und vereint Wissenschaftliche (,sciences et patrimoine') wie Nicht-wissenschaftliche (Schulbibliotheken und ,lecture publique') Bibliotheken des Kantons. ${ }^{38}$

Die Westschweizer Erziehungsdirektorenkonferenz hat in dieser Situation eine Übergangsstruktur für die Leitung des Réseau romand bis zum Jahr 2019 geschaffen und ein Steuerungsgremium (Comité de pilotage) mandatiert, das vor dem Hintergrund der eingetretenen Veränderungen zusammen mit der Verbunddirektion eine Strategie für die Zukunft von RERO nach dem Jahr 2019 und ein zukunftsfähiges Geschäftsmodell erarbeitet. Denn durch den Austritt des Kantons Waadt und das Projekt SLSP, an dem die Hochschulen der übrigen Westschweizer Kantone (also die Universitäten Genf und Freiburg, die Westschweizer Fachhochschule sowie künftig die Universität Neuenburg) teilnehmen bzw. teilnehmen werden, stellt sich die Tätigkeitsgrundlage von RERO grundlegend verändert dar. Vor dem Hintergrund der neuen Ausgangslage wird es einerseits darum gehen,

37 Siehe den Beitrag „,Gemeinsam sind wir stärker““ von Wolfram Neubauer in diesem Band. Die Erwartungen der Community zeigen beispielhaft Karsten Schuldt und Rudolf Mumenthaler: Vor welchen Veränderungen steht die schweizerische Verbundslandschaft? In: 027.7 Zeitschrift für Bibliothekskultur 2/1 (2014), S. 5-11 (http://dx.doi.org/10.12685/027.7-2-1-47).

38 www.bcu-lausanne.ch/renouvaud/renouvaud-historique. Siehe auch Rapport annuel 2016 de la BCU Lausanne, S. 44-46 (www.bcu-lausanne.ch/wp-content/uploads/2017/06/BCUL-RAP PORT-ANNUEL-2016.pdf). Die ausführliche Projektbeschreibung (Rapport d'initialisation) ist unveröffentlicht. 
erfolgreiche Dienstleistungen wie z.B. das konsortial organisierte Dokumentenrepository RERO-DOC oder das Hosting der Plattform digitalisierter Schweizer Presse fortzuführen und andererseits neue Dienstleistungen für einen Kundenkreis zu schaffen, der durch das auf die Wissenschaftlichen Bibliotheken ausgerichtete Projekt SLSP nicht bedient wird. ${ }^{39}$ Hierzu laufen die Abklärungen; so hat RERO angekündigt, ein schlank angelegtes, auf die Bedürfnisse kleinerer Bibliotheken skalierbares Open Source-Bibliotheksverwaltungssystem als Software as a service-Lösung anzubieten. ${ }^{40}$

Neben den genannten grossen Verbünden existiert noch eine Zahl kleiner regionaler, von Kantonsbibliotheken betriebener Verbünde, so u. a. in der italienischsprachigen Schweiz, in Graubünden, im Thurgau, im Kanton St. Gallen und im Aargau, sowie ein Verbund der Bibliotheken der Bundesverwaltung. ${ }^{41}$ Zunächst werden die universitären Bibliotheken und, soweit sie in den genannten Verbundwelten IDS oder RERO mit ihren Daten vertreten sind, die Bibliotheken der Fachhochschulen und Pädagogischen Hochschulen auf die neue Serviceplattform wechseln ebenso wie die Mehrzahl der Spezialbibliotheken und Bibliotheken hochschulunabhängiger Forschungseinrichtungen, die schon heute Mitglieder einer dieser Verbundwelten sind. Die übrigen Bibliotheken mit wissenschaftlichem Charakter, das heisst konkret: Insbesondere die kleineren Kantonsbibliotheken vor allem in den Deutschschweizer Kantonen, die heute in unabhängigen Regionalverbünden arbeiten oder diese gar selbst betreiben, werden sich zu einem späteren Zeitpunkt ebenfalls der neuen Plattform anschliessen können, sobald die Erstmigrationen erfolgreich bewältigt sind und die Swiss Library Service Platform ihren regulären Betrieb aufgenommen hat.

39 Allerdings will auch SLSP den Kantonsbibliotheken mit wissenschaftlich relevanten Sammlungen mittelfristig seine Dienstleistungen öffnen.

40 Proposition d'un centre de compétences et de services aux bibliothèques suisses, veröffentlicht am 27.10.2016: www.rero.ch/page.php?section=infos\&pageid=centre-competences-fr-20161027.

41 Sistema bibliotecario ticinese (www.sbt.ti.ch), Bibliotheksverbund Graubünden (www.kan tonsbibliothek.gr.ch), Thurgauer Bibliotheksverbund (www.biblio.tg.ch), St. Galler Bibliotheksnetz (www.sgbn.sg.ch), Aargauer Bibliotheksnetz (www.ag.ch/de/bks/kultur/archiv_bibliothek/ kantonsbibliothek/bibliotheksnetz/abn.jsp). Die Bibliotheken der Bundesverwaltung sind im Bibliotheksverbund Alexandria (www.alexandria.ch) organisiert. 


\section{Kooperations- und Netzwerkstrukturen auf dem Weg zur Institutionalisierung}

Neben den klassischen Bibliotheksverbünden müssen in diesem Überblick noch weitere Kooperations- und Netzwerkstrukturen Erwähnung finden, die sich teils auf dem Weg zur Institutionalisierung befinden, teils bereits einen fest etablierten Status erreicht haben. Einige davon verdanken ihre Entstehung der ersten Welle von Bundesfördergeldern, mit der das Rahmenprojekt e-lib.ch: Elektronische Bibliothek Schweiz in den Jahren 2008-2013 ermöglicht wurde; ${ }^{42}$ seit dessen Auslaufen haben sich einige der dort initiierten Projekte verstetigen können.

Für die Versorgung der Schweizer Hochschulen mit elektronischen Publikationen erbringt bereits seit dem Jahr 2000 das Konsortium der Schweizer Hochschulbibliotheken Dienstleistungen, die von allen Teilnehmern als unentbehrlich eingestuft werden. ${ }^{43}$ Durch eine Anschubfinanzierung der Schweizerischen Universitätskonferenz auf den Weg gebracht, arbeitet es heute selbsttragend. Die grosse Mehrzahl der von den Wissenschaftlichen Bibliotheken erworbenen E-Medien-Lizenzen sowohl im Zeitschriften- und Datenbanken- als auch zunehmend im E-Book-Bereich wird konsortial verhandelt, was eine weitgehende Einheitlichkeit der Lizenzbedingungen sicherstellt und Nachteilen für die lizenznehmenden Bibliotheken bei Preisen und Konditionen vorbeugt, die durch eine unabgestimmte Verhandlungsführung entstehen könnten. Die Lizenzkosten selbst sind jedoch nicht (auch nicht anteilig) zentral finanziert. Das Konsortium hat im Jahr 2016 mit Förderung durch die projektgebundenen Beiträge der Schweizerischen Hochschulkonferenz ${ }^{44}$ Nationallizenzen für Zeitschriftenarchive von vier grossen Wissenschaftsverlagen abgeschlossen, wobei es gelungen ist, die Öffnung auch für registrierte Privatpersonen ohne Hochschulzugehörigkeit mit Wohnsitz in der Schweiz zu erreichen. Neben verschiedenen die Lizenzierung ergänzenden Dienstleistungen für die teilnehmenden Bibliotheken - etwa die Bereitstellung von Nutzungsstatistiken und Support im Management des Zugangs zu den Verlagsinhalten - ist als weiteres Themenfeld die Langzeitarchivierung der über Konsortialverträge erworbenen Inhalte hinzugekommen, wofür übergreifende Lizenzverträge mit den Diensten Portico und LOCKSS geschlossen

42 Mehr dazu im Beitrag „Nationale Förderprogramme“ von Alice Keller in diesem Band sowie bei Wolfram Neubauer: Schweizer Bibliotheken im Netz: Stand und Zukunft digitaler Angebote. In: Bibliothek - Forschung und Praxis 36 (2012), S. 69-76.

43 Ausführlicher vorgestellt im Beitrag „Konsortium“ von Rafael Ball und Pascalia Boutsiouci in diesem Band.

44 Vgl. dazu Anm. 29. 
wurden. Aktuell engagiert sich das Konsortium in der Entwicklung der Umsetzungsplanung für die nationale Open Access-Strategie, die vom Staatssekretariat für Bildung, Forschung und Innovation, der Rektorenkonferenz swissuniversities und dem Schweizerischen Nationalfonds zur Förderung der wissenschaftlichen Forschung (SNF) initiiert wurde. Mittelfristig wird angestrebt, die Dienstleistungen des Konsortiums, das derzeit noch projektförmig innerhalb der ETH Zürich organisiert ist, in die neue Serviceplattform SLSP $\mathrm{zu}$ integrieren und seine Dienstleistungen im Zusammenspiel mit dem neuen zentralen Bibliotheksverwaltungssystem auch auf die operative Verwaltung der konsortial lizenzierten Produkte auszuweiten.

Bereits als eigenständige Institution etabliert hat sich die Anfang 2016 eröffnete Kooperative Speicherbibliothek Schweiz mit Standort in der Zentralschweiz, im Kanton Luzern. ${ }^{45}$ Entstanden auf eine Initiative aus der Zentral- und Hochschulbibliothek Luzern hin, haben sich hier grosse Bibliotheken und mehrere Kantone zusammengefunden, um für ihre magazinierten Bestände die Platzprobleme, die an den Standorten in den Innenstädten bestehen, durch eine mit moderner Logistiktechnik ausgerüstete, von einem durch alle Partner getragenen Verein in zentraler geographischer Lage betriebene Speicherbibliothek zu lösen. Ein Grundelement der bibliothekarischen Konzeption ist dabei ein gemeinsames, auf ein Exemplar pro Titel verdichtetes Archiv von Printzeitschriften, wobei das Eigentum am Bestand auf den Trägerverein übertragen wurde, der die langfristige Aufbewahrung und Zugänglichmachung garantiert.

Die Speicherbibliothek arbeitet kostendeckend und wird von den Partnern gemäss ihrem Anteil am Magazinraum und den in Anspruch genommenen Dienstleistungen finanziert. In einer Doppelkonstruktion einer Aktiengesellschaft, der die Immobilie gehört und deren Aktionäre die Hochschulen bzw. Kantone sind, ${ }^{46}$ und einem Betriebsverein, in dem alle Partner Mitglieder sind und der Beiträge bei den Bibliotheken erhebt, wurde eine stabile und gleichzeitig von sich ändernden politischen Verhältnissen weitgehend unabhängige rechtliche Form gefunden. Die Erweiterung des Teilnehmerkreises ist möglich, denn die Speicherbibliothek verfügt über Grundstücksreserven für weitere Lagermodule. Da sie sich ausschliesslich als Dienstleisterin für ihre Bibliotheken versteht, hat sie kein eigenes aktives Bestandskonzept. Die Existenz des gemeinsamen Zeitschriftenarchivs garantiert darum noch nicht die Weiterführung der Zeitschrif-

45 Zur Speicherbibliothek vgl. auch den Beitrag „Der weite Blick“ von Ulrich Niederer in diesem Band.

46 Als öffentliche Stiftung ist die Zentralbibliothek Zürich eine selbstständige Rechtspersönlichkeit und tritt darum selbst in der Aktiengesellschaft Speicherbibliothek als Aktionärin auf. 
ten - die Speicherbibliothek nimmt zwar die aktuellen Jahrgänge auf und fügt sie dem kollektiven Bestand hinzu; die Bibliotheken sind aber frei, Titel abzubestellen. In den kommenden Jahren wird das Potenzial für eine Kooperation mit der neuen Serviceplattform SLSP in den Bereichen Logistik, Archivierung und Dokumentlieferdienste zu klären sein; erste Sondierungen dazu haben stattgefunden. Die Speicherbibliothek könnte im Zuge dieser Entwicklung ebenfalls in die Rolle eines nationalen Dienstleisters hineinwachsen.

Im schon erwähnten Förderprogramm e-lib.ch haben die ebenfalls schweizweit ausgerichteten digitalen Plattformen für Alte Drucke und Rara (e-rara.ch), für neuzeitliche und mittelalterliche Handschriften (e-manuscripta.ch, e-codices) und für Schweizer wissenschaftliche Zeitschriften (E-Periodica) ihren Ursprung. Weitere Plattformen präsentieren u.a. kartographische Materialien (Kartenportal. $\mathrm{CH}$ ), Bildquellen (E-Pics der ETH-Bibliothek) und Zeitungen (Schweizer Presse Online, koordiniert von der Schweizerischen Nationalbibliothek in technischer Zusammenarbeit mit RERO). Sie werden in diesem Band einzeln präsentiert; ${ }^{47}$ an dieser Stelle sei nur vermerkt, dass die Plattformen für Alte Drucke e-rara.ch und für neuzeitliche Handschriften e-manuscripta.ch sich 2016 einer Open Data-Policy verpflichtet haben, die die wissenschaftliche Nachnutzung der Metadaten und der Inhalte erleichtern und so die Forschungsrelevanz der digital bereitgestellten Quellen erhöhen will. Auch weitere aktuelle Bestrebungen zum Ausbau der Plattformen richten sich auf die optimale Forschungsunterstützung bei der Nutzung der angebotenen Materialien, so z.B. durch Transkriptions- und Annotationswerkzeuge oder die Öffnung für Text Mining-Verfahren durch den Einsatz hochperformanter, an die Besonderheiten des Materials angepasster Texterkennungs- und Indizierungsapplikationen. Schliesslich wird die Integration internationaler Interoperabilitätsstandards für digitale Objekte wie etwa des IIIFFrameworks und die Implementierung dazugehöriger Standardschnittstellen vorbereitet, um den Anschluss der Schweizer Plattformen des kulturellen Erbes an europäische und globale Anwendungs- und Präsentationskontexte zu gewährleisten.

Schliesslich ist der Metakatalog der Schweizer Bibliotheken swissbib zu erwähnen, dessen Aufbau seit 2008 ebenfalls durch Förderung im Rahmen von e-lib.ch und durch das Nachfolgeprogramm „Wissenschaftliche Information“ ermöglicht wurde. ${ }^{48}$ Das Projekt und der Betrieb sind an der Universitätsbibliothek

47 In den Beiträgen des Teils „Initiativen zu historischen Sammlungen und Spezialbeständen“ in diesem Band.

48 In diesem Band vorgestellt im Beitrag „Swissbib“ von Günter Hipler, Nicolas Prongué und René Schneider. 
Basel angesiedelt. Swissbib sammelt die Katalogdaten der Schweizer Verbünde, der Nationalbibliothek und weiterer Einzelinstitutionen in einem normalisierten Index auf einer eigenen Datenplattform und macht sie über ein Suchinterface zugänglich. Die aufbereiteten Daten werden im Auftrag der IDS-Verbünde durch swissbib auch an OCLC WorldCat geliefert. Da RERO ebenfalls mit WorldCat kooperiert, sind seit 2010 die Schweizer Bibliotheksdaten mehrheitlich auch über diesen globalen Datenhub nachgewiesen.

Swissbib hat aufgrund seiner breiten Datenabdeckung, der modernen und intuitiven Suchoberfläche und der Integration von Benutzungsfunktionen für die lokalen Bestände über Webservices einen hohen Bekanntheitsgrad bei den Benutzern erlangt. Aktuell stellt die Universitätsbibliothek Basel als Projektträgerin Überlegungen an, welche Aufgaben sich für swissbib nach dem Start der neuen gemeinsamen Datenplattform von SLSP stellen. SLSP wird eine zentrale Discovery-Lösung anbieten, welche freilich zumindest zu Beginn nicht alle heute über swissbib nachgewiesenen Datenbestände (Nationalbibliothek, Kantonsbibliotheken, Renouvaud) enthalten dürfte. Swissbib stellt seinen Index über eine API auch als Linked Open Data im RDF-Format zur Verfügung und hat dadurch im Gegenzug den eigenen Suchraum mit normierten Datenbeständen wie DBpedia und der internationalen Normdatenplattform VIAF anreichern können. Über das dazu von swissbib gemeinsam mit den Fachhochschulen in Genf und Chur durchgeführte Projekt linked.swissbib.ch wird in diesem Band berichtet.

Während diese Ausführungen zeigen, dass das Potenzial, das national angelegte Kooperationen in einem kleinen Land wie der Schweiz im digital vernetzten Umfeld bieten, seit Längerem erkannt und in exemplarischen Projekten auch realisiert wurde - Projekten, deren Verstetigung in stabilen, nachhaltig finanzierten Services teilweise bereits gelungen ist -, ist die institutionelle Basis für ein auf Dauer angelegtes kooperatives Vorgehen bisher noch schmal: Hier zeigt sich noch die bereits anfangs angesprochene stark föderalistische Prägung des Wissenschafts- und Kultursektors in der Schweiz. Als eines der wenigen landesweit angelegten Gremien im bibliothekarischen Bereich hat sich die Konferenz der Universitätsbibliotheken (KUB/CBU) der Koordination übergreifender bibliothekarischer Sachfragen, immer stärker aber auch der strategischen Abstimmung zwischen den Hochschulbibliotheken verpflichtet ${ }^{49}$ - aktuell etwa zur Umsetzung der nationalen Open Access-Strategie und deren Konsequenzen für die Lizenzierung oder bei der Debatte um die Erneuerung des schweizerischen Urheberrechts. Auch tritt sie als Auftraggeberin des Konsortiums auf. Dennoch verfügt sie bisher

49 Zur KUB siehe auch den Beitrag „Nationale Förderprogramme“ von Alice Keller in diesem Band. 
weder über eine klare institutionelle Einordnung noch über ein formelles Mandat mit normativer Qualität für den Hochschulbibliothekssektor. Um diese Unschärfe zu überwinden, wird aktuell diskutiert, in welcher Form die KUB in die Strukturen der Schweizerischen Hochschulrektorenkonferenz swissuniversities integriert oder zumindest enger an diese angebunden werden kann.

\section{Konvergenz der Handlungsfelder in der wissenschaftlichen Informationsinfrastruktur - neue Konstellationen der Akteure}

Was bringt die Zukunft für das wissenschaftliche Bibliothekswesen der Schweiz? Ganz konkret ist ein grosser Veränderungsimpuls vom schon verschiedentlich gestreiften Projekt Swiss Library Service Platform (SLSP) zu erwarten, auf dem grosse Hoffnungen für die Etablierung einer dauerhaften nationalen Kooperationsstruktur ruhen. Vordergründig geht es um den Umstieg auf eine cloudbasierte Bibliotheksverwaltungslösung der neuen Generation. Tatsächlich ist der Anspruch aber viel umfassender: An die Stelle regional organisierter Verbünde klassischen Typs wird eine von den Bibliotheken getragene zentrale Serviceagentur treten, die über die mit einem neuen Bibliothekssystem verbundenen Angebote hinaus umfassende digitale und prozessunterstützende Dienstleistungen gemäss Nachfrage und Bedarf der Bibliotheken entwickeln und dann zentral und kostendeckend betreiben wird. Die Vermittlung weiterer Dienste, die von einzelnen Bibliotheken oder auch Dritten aufgebaut, aber allen SLSP-Kunden angeboten werden, kann hinzutreten. SLSP wird, so die Erwartung, auch in internationalen Kontexten zur Stimme der Schweizer Bibliotheken werden und so Sichtbarkeit und Gewicht der hiesigen Infrastrukturen des Bibliotheks- und Informationssektors in Entwicklungen erhöhen, die über die Schweiz hinausreichen.

Ausschlaggebend für die Relevanz der Wissenschaftlichen Bibliotheken im Hochschulumfeld dürfte in der vor uns liegenden Periode werden, ob es ihnen gelingt, von der Forschungscommunity akzeptierte Dienstleistungen zur Unterstützung des (immer häufiger auch kollaborativ organisierten) Forschungsprozesses und zu einer umfassenden Dokumentation, Präsentation und Nachnutzung von Forschungsergebnissen zu entwickeln. Dabei sind sich die Akteure bewusst, dass sich der Bibliotheksbereich angesichts der technologiegetriebenen Konvergenzen im Zuge der digitalen Transformation von Wissenschaft und Forschung nicht isoliert positionieren darf, sondern sich als Teil einer umfassenden Infrastrukturlandschaft der wissenschaftlichen Information verstehen muss. Das Ausloten des Kooperationspotenzials mit sich etablierenden Anbietern und Netzwer- 
ken im Feld der wissenschaftsunterstützenden IT (Scientific Computing, Science IT) hat begonnen und gibt erste Hinweise auf künftig mögliche gemeinsame Dienstleistungen. So wird im Rahmen des Projekts SLSP mit der von den Hochschulen, Bund und Kantonen getragenen Stiftung SWITCH, die zentral IT-Netzwerkdienste für den Schweizer Hochschulraum zur Verfügung stellt, über die Anbindung der Benutzerverwaltung der neuen Plattform an die Identitätsmanagementdienste von SWITCH nachgedacht. ${ }^{50}$ Auch Hosting- und Cloud Computing-Services von SWITCH sind für die Bibliotheken von Interesse und werden in einigen Innovationsprojekten bereits genutzt. ${ }^{51}$ Schliesslich erscheint längerfristig auch eine organisatorische Beziehung zwischen beiden Anbietern vorstellbar.

Darüber hinaus zeichnen sich bei einer Reihe von strategisch wichtigen Themen Netzwerkstrukturen ab, die Bibliotheken, Informatikdienste der Hochschulen und ihre forschungsunterstützenden IT-Services als komplementäre Akteure zusammenbringen: Dies gilt etwa für die digitale Langzeitarchivierung, ${ }^{52}$ die föderierte Speicherung und Bereitstellung von Forschungsdaten einschliesslich eines rollenbasierten Rechtemanagements, die Unterstützung von ,Collaborative Science'-Strukturen und generell für die Herausforderung, den gesamten Forschungszyklus zu begleiten und Forschungsoutput in einem umfassenden Sinn - also über das Resultat, das in einem klassischen Publikationsformat seinen Niederschlag findet, hinaus - verfügbar zu machen. Freilich ist heute noch kaum verlässlich einzuschätzen, ob und in welcher Weise dies zu dauerhaften betrieblichen und organisatorischen Strukturen führen wird..$^{53}$

50 Die Überlegung geht dahin, dass die Stammdaten der Benutzer ausserhalb des Bibliothekssystems verwaltet werden, nämlich in der AAI-basierten Identitätsmanagementlösung SWITCH edu-ID (vgl. dazu den Beitrag „Digitale Identitäten“ von Christoph Graf in diesem Band), während im Bibliothekssystem idealerweise nur die Transaktionsdaten liegen. Die Verknüpfung würde über die edu-ID-Nummer erfolgen. Es bleibt abzuklären, ob eine solche Lösung mit dem künftigen Bibliotheksverwaltungssystem von SLSP technisch möglich ist.

51 So wird der Hostingdienst SWITCHengines vom Schweizer Geodatenportal für Lehre und Forschung geodata4edu.ch (vgl. dazu den Beitrag „Der nationale Service für Geodaten“ von Arlette Piguet in diesem Band) für Webservices zur Weiterverarbeitung der Daten und für den Betrieb der Suchmaschine eingesetzt. Die Universitätsbibliothek Bern betreibt ihr OAIS-konformes digitales Archiv mithilfe von SWITCHengines (dazu Marion Prudlo: Schritt für Schritt zum Langzeitarchiv. In: SWITCH Journal März 2016, www.switch.ch/de/stories/library_archive).

52 Vgl. dazu in diesem Band den Beitrag von Pierre Yves Burgi und Eliane Blumer über das Projekt Data Life-Cycle Management (DLCM), das Tools und Ressourcen, Best Practice-Beispiele und Lehrmaterialien zur digitalen Langzeitarchvierung zur Verfügung stellt.

53 Im Jahr 2017 haben die beiden Eidgenössischen Technischen Hochschulen in Zürich und Lausanne gemeinsam das Swiss Data Science Center gegründet, um ,Datenwissenschaft‘ in Ausbildung und Forschung zu etablieren (u.a. durch den Aufbau eigener Studiengänge), den Wissenstransfer zu beschleunigen und disziplinübergreifende Infrastrukturen aufzubauen, die 
Das Beispiel der erst entstehenden Strukturen für das Forschungsdatenmanagement führt ausserdem zu der Frage, welche Rolle disziplinäre Serviceverbünde künftig spielen werden. Generische Services können häufig nur ein begrenztes Potenzial entfalten, wenn die spezifischen disziplinabhängigen Anforderungen sehr ausgeprägt sind, die sich aus der Art der Daten, der eingesetzten Forschungsinstrumente, der Publikationserfordernisse oder der Nachnutzungsbedingungen ergeben. In einigen Fachkontexten, so in den empirischen Sozialwissenschaften ${ }^{54}$ oder den Kunstwissenschaften ${ }^{55}$, zeichnen sich bereits starke disziplinspezifische Infrastrukturen ab. Gerade im natur- und lebenswissenschaftlichen Bereich sind diese Kontexte in der Regel auch gar nicht mehr national und institutionell definiert, vielmehr sind (oft ungesteuert) virtuelle föderierte Servicenetze mit transnationaler Reichweite entstanden, die eine hochspezialisierte, aber weltweit verteilte Forschungscommunity verbinden. Ob es den Bibliotheken - bei allem Ausgreifen in den virtuellen Raum - gelingt, hier eine systemrelevante Rolle einzunehmen, wird man mit Vorsicht bewerten müssen.

Wissenschaftspolitisch ist in jüngster Zeit auch in der Schweiz zu beobachten, dass die strategische Bedeutung der Entwicklung der Informationsinfrastrukturen vom Wissenschaftsmanagement und von den Hochschulleitungen erkannt worden ist. Die Steuerung von Handlungsfeldern, deren kritische Systemrelevanz die Bibliotheken bis vor Kurzem ihren institutionellen und politischen Trägern

auch den übrigen Akteuren im Schweizer Hochschulraum zur Verfügung stehen sollen. Adressiert werden Themen wie die verteilte Prozessierung grosser Datenmengen (,Big and Fast Data') oder maschinelles Lernen mit statistischen Methoden. Als Service wird eine cloudbasierte Datenanalyseplattform vorgehalten. Die Hochschulbibliotheken sind an dieser Initiative bisher nicht beteiligt (worden) - sie sollten umso mehr klären, welche Angebote und Kompetenzen sie einbringen können.

54 In den empirischen Sozialwissenschaften konnte sich seit 2008 FORS als nationales Kompetenzzentrum mit einem Dienstleistungsschwerpunkt in der Datenhaltung und -distribution erfolgreich positionieren. In den Geistes- und Kulturwissenschaften soll sich das seit 2017 von der Schweizerischen Akademie für Geistes- und Sozialwissenschaften geführte Data and Service Center for the Humanities (DaSCH) als Betreiber einer generischen Forschungsdatenplattform und Dienstleister für Beratung und Schulung zu Standards, Technologien und Best Practice etablieren. Es wird sich erweisen, welche Bindungskraft ein solches generisches Serviceangebot in den kleinteilig organisierten und durch Methodenvielfalt gekennzeichneten geisteswissenschaftlichen Fächern in der Praxis zu entwickeln vermag. Zum DaSCH siehe den Beitrag von Beat Immenhauser in diesem Band.

55 In der Startphase befindet sich die Swiss Art Research Infrastructure (SARI), die als Teil des Linked Open Data-Netzes Teil einer internationalen fachlichen Forschungsinfrastruktur werden soll: www.khist.uzh.ch/de/chairs/neuzeit/res/sari.html. SARI findet sich auf der Schweizer Roadmap für Forschungsinfrastrukturen von nationaler Bedeutung 2017-2020 des Staatssekretariats für Bildung, Forschung und Innovation (SBFI). 
nicht ohne Weiteres vermitteln konnten, wird nun häufig von den institutionellen Stakeholdern selbst beansprucht: Das hängt ohne Zweifel mit der erwähnten Konvergenz der Infrastrukturbereiche Bibliothek, IT-Services und Scientific Computing infolge der digitalen Transformation der Hochschulen und Forschungseinrichtungen und den dafür benötigten erheblichen Ressourcen zusammen. Sowohl für den Aufbau einer umfassenden digitalen Infrastruktur für Forschung und Lehre als auch für die flächendeckende Beschaffung bzw. Aufbereitung digitaler Inhalte werden finanzielle Mittel in einer Grössenordnung erforderlich, die eine zentrale strategische Steuerung aus Managementsicht zum unbedingten Imperativ macht.

In dieses Bild passt die Lancierung der grossen Förderprogramme durch den Bund und die Unterstützung einer Konsolidierung bisher kleinteiliger Strukturen, wie sie die Bibliotheken exemplarisch mit dem Aufbau der zentralen Serviceplattform SLSP angehen. Auch dort engagieren sich die Hochschulen mit ihrer strategischen Leitungsebene direkt und investieren mit dem Erwerb von Anteilen an der neugegründeten nicht-gewinnorientierten Aktiengesellschaft und der Gewährung von Darlehen in substantieller Höhe. Eine nationale Open Access-Strategie, um ein letztes Beispiel zu nennen, hätte ohne Aufnahme in die wissenschaftspolitische Agenda - und zwar auf einer hohen Lenkungsstufe, flankiert durch gleichgerichtete Positionierungen der Europäischen Kommission - und als Forderung der Bibliotheken allein kaum die Schubkraft entfaltet, die nun mit der ambitionierten politischen Zielsetzung festzustellen ist, die Ergebnisse der in der Schweiz öffentlich geförderten Forschung bis zum Jahr 2024 vollständig im Open Access zugänglich zu machen.

Aus dem bis 2020 laufenden aktuellen Förderprogramm „Wissenschaftliche Information“ (P-5) soll nach Vorstellung der Träger eine auf Dauer angelegte Koordinationsplattform erwachsen, die als ,Servicedrehscheibe“ die Kohärenz der geförderten Projekte stärkt, ihren Übergang in stabilisierte Dienste unterstützt, deren Durchlässigkeit und Offenheit für alle Wissenschaftseinrichtungen sicherstellt und neue Trends und Entwicklungen im Feld der wissenschaftlichen Informationsinfrastrukturen in den Förderkontext einspielt und den Akteuren zuordnet. $^{56}$ Die Konstruktion zielt darauf ab, „Synergien in der Entwicklung und Benutzung von Dienstleistungen im Bereich des wissenschaftlichen Arbeitens mit digitaler Information [zu] schaffen“". ${ }^{57}$ Es ist also nicht vorgesehen, dass diese

56 Mehr dazu im Beitrag „Das Programm P-5“ von Gabi Schneider in diesem Band.

57 Grobkonzept Nationale Organisation, S. 7 (www.swissuniversities.ch/de/organisation/pro jekte-und-programme/p-5/projekt-nationale-organisation). Dort finden sich auch weitere Ausführungen zu den anvisierten Aktivitäten der Koordinationsstelle. Das Konzept wird derzeit genauer ausgearbeitet. 
Stelle selbst Dienstleistungen erbringt - dies soll weiterhin den Hochschulen und den in ihrem Auftrag und Organisationsbereich handelnden Akteuren vorbehalten sein.

Auch wenn im Augenblick noch nicht feststeht, welche Form diese Koordinationsstelle erhalten und wie ihre Zuständigkeit konkret ausgestaltet wird, so steht doch fest, dass die Bibliotheken ein existentielles Interesse haben, in diese entstehende Struktur einbezogen zu sein und ihre Bedürfnisse ebenso wie ihre Leistungsangebote dort einzubringen. Im Hintergrund der Bemühungen um eine solche Koordinationsstruktur, die auf nationaler Ebene in der Schweiz bisher fehlte, ${ }^{58}$ steht die mit dem Hochschulförderungs- und -koordinationsgesetz seit dem Jahr 2017 für den Bund geschaffene Möglichkeit, Infrastrukturen der Hochschulen auch dauerhaft im Betrieb zu fördern. ${ }^{59}$ Wie gesagt, es handelt sich um eine rechtliche Möglichkeit, zu deren wirksamer Realisierung es stets den politischen Willen und ein günstiges öffentliches Meinungsbild benötigen wird, für das die Bibliotheken durch eine Konzentration ihrer Kräfte eine wichtige Voraussetzung schaffen können: zum Aufbau innovativer Lösungen, die den Anforderungen der Wissensgesellschaft an leistungsfähige Dienstleistungseinrichtungen für Bildung, Wissenschaft und Kultur genügen.

58 Die Situation ist in dieser Hinsicht bisher grundlegend anders als z. B. in Deutschland, wo mit der Zuständigkeit u.a. der Deutschen Forschungsgemeinschaft (DFG) für die Infrastrukturförderung im Bereich der Wissenschaftlichen Bibliotheken und Informationseinrichtungen seit vielen Jahrzehnten eine solche Koordination der Förderung von Informationsinfrastrukturen besteht: Förderziel ist „der Aufbau leistungsfähiger Informationssysteme für die Forschung unter überregionalen Gesichtspunkten“ (www.dfg.de/lis). Während bis vor wenigen Jahren mit der Förderung der sog. Sondersammelgebiete auch eine langfristige Finanzierung des Bestandsaufbaus ausgewählter Bibliotheken gemäss einem überregionalen Fächerverteilungsplan dazu gehörte, leistet die DFG heute überwiegend Anschubfinanzierungen für innovative Dienste zur Forschungsunterstützung, dies freilich über ein breites Themenspektrum hinweg. In Deutschland hat auch der Bund seit Langem Institutionen und Projekte der wissenschaftlichen Fachinformation, vor allem im technisch-naturwissenschaftlichen Bereich, (ko-)finanziert und ist über die Leibniz-Wissenschaftsgemeinschaft auch Träger der sog. Zentralen Fachbibliotheken für Wirtschaft, Technik und Naturwissenschaften.

59 HFKG Art. 47 Abs. 3: „Der Bund kann Finanzhilfen in Form von Beiträgen an gemeinsame Infrastruktureinrichtungen der Hochschulen und der anderen Institutionen des Hochschulbereichs gewähren, wenn die Infrastruktureinrichtungen Aufgaben von gesamtschweizerischer Bedeutung erfüllen. Diese Beiträge betragen höchstens 50 Prozent des Betriebsaufwandes.“ (SR 414.20, wie Anm. 10). 
